SULLE SCARICHE INTERNE DEI CONDENSATORI ELETTRICI; HICERCHE DEL PROF. EMLLIO VILLART. (QUARTA MEMORIA).

Quando (") una batteria di bottiglie di Leyda fortemente carica, si scarichi per mezzo di uno dei miei termometri ec. citatori, in modo che la scintilla eccitatrice unica si formi nel suo interno e faccia così sentire poco strepito, riesce cosa assai facile di avvertire un rumore sordo, e come un tonfo, prodursi nella batteria. E se si fanno le osservazioni nel buio, le bottiglie ai bordi delle loro armature si vedranno, nell'istante della scarica, vivamente illuminate da sprazzi di luce violacea, fitti ed arborescenti, che si distendono sul lembo verniciato del vetro. Codesti fenomeni crescono in modo assai rapido col crescere delle cariche dei condensatori adoperati. Dall'altro canto se si studia il calore svolto dalla scintilla eccitatrice si scorge, che esso cresce rapidissimamente con le cariche tino a un certo limite, coincidente presso a poco col manifestarsi del tonfo, e poscia cresce assai più lenta-

(1) Di parte di questo lavoro fu dato un cenno in una Nota inserita nei R-C del R. I. Lombardo, 29 Luglio 1880. 
mente (1): sul quale limite, forse ha molta influenza la natura delle bottiglie, come dirò in una prossima occasione.

Questi ed altri fatti consimili, da me più volte notati, mi fecero credere che scaricando un condensatore fortemente carico dovesse, oltre la scarica ordinaria, generarsene una nello stesso condensatore, con produzione non solo di luce ma eziandio di colore. Per verificare tale supposizione costruii uua specie di grosso termometro a gasse T'T" (Tav. I. fig. 1) formato da un'ampia canna di vetro contenente una bottiglia di Leyda B, lunga $50 \mathrm{~cm}$. larga $10 \mathrm{~cm}$; ricoperta per due terzi di stagnola ed il resto accuratamente rivestito di vernice a gommalacca e spirito. Lo aperture della canna son chiuse da ghiere di ottone stuccate diligentemente con mastice, così da tener l'aria. La inferiore delle quali $\mathrm{T}^{\prime \prime}\left({ }^{2}\right)$ (stando la canna verticale) sostiene la bottiglia, appoggia sopra un panchetto isolante $\mathrm{PP}^{\prime}$ ed è unita ad una bottiglia elettrometrica $\mathrm{F}$, che serve a misurare lo cariche comunicate alla bottiglia del termometro. La ghiera superiore $\mathrm{T}$ porta nel centro saldato un lungo tubo di ottone $t$, che con l'un dei capi penetra nella bottiglia e con tre forti molle di ottone ad esso saldato preme e comunica con l'armatura interna di quella. Il capo esterno e superiore $t$ è unito, con un tubo di gomma $t t$ ad un cannello di vetro $t^{\prime} v$; immerso con l'estremità libera nel vaso di vetro $v$ contenente alcool, del quale una lunga colonna penetra nel cannello, $\theta$ coi suoi movimenti manifesta lo variazioni di volume dell aria contenuta nel termometro a gasse. I movimenti dell'indice si osservano, ad occhio nudo se estesi, e so piccoli si guardano con un cannocchiale: ed essi

(1) In una breve Nota, Villari, Rendiconto d. A. d. S. di Bologna, pag. 143 (1879-80) „ jo ho detto che il calore svolto dalla scintilla eccitatrice d' un condensatore è proporzionale, in modia, al quadrato dolle caricbe, nei limiti delle mie esperienze. Nel lavoro completo, che pubblicherò fra poco, su questo soggetto trattero estesamente una tale questione ed indicherò esattamente $i$ limiti nei quali detta legge si riscontra.

(2) In questa prima disposizione dell'apparecchio la ghiera ' $T$ formava il fondo del tormometro nell'interno del quale era contenuta la bottiglia B: in seguito l'apparato fu modificato ael modo indicato nella figura 1. 
si misurano su di una scala divisa in millimetri ed addossata al cannello. Finalmente allo stesso tubo $t$ è saldata l'asta di rame $n n^{\prime}$ terminata a palline, che serve a caricare e scaricare facilmente la bottiglia.

Con codesto apparecchio eseguii alcune misure nel modo seguente. Caricavo fortemente con una macchina Holtz la bottiglia $\mathrm{B}$, poscia la scaricavo con l'eccitatore $e e^{\prime}$ ed osservavo col cannocchiale l' indice del cannello $t^{\prime} v$; il quale indice nel momento della scarica s' abbassava, dinotando una dilatazione dell'aria nel termometro, che pareva generata da riscaldamento. Temendo che un simile effetto potesse esser prodotto da scintille scattanti fra le armature della bottiglia $\theta$ le altre parti metalliche con cui quelle erano in contatto, cercai di impedire assolutamente la formazione di tali supposte scintille nell' interno del termometro. Per la qual cosa masticiai da prima la bottiglia $B$ all' orlo inferiore $T^{\prime \prime}$ della canna, così che l'armatura esterna della bottiglia rimaneva in parte fuori della canna, ed appoggiava direttamente sul piatto metallico $T$, il quale comunicava poi con la bottiglia elettrometrica E. Ad evitar poi le scintille che scattar potessero fra il conduttore $\mathrm{B} t$ e l' armatura interna, forzai energicamente le tre molle d'ottone, unite al detto conduttore e le ficevo con gran forza pigiare contro l'armatura: in alcuni casi inoltre, come dirò più ampiamente in seguito, riempivo per due terzi la bottiglia di mercurio, il quale faceva da armatura interna, ed immergevo in esso un tubo di rame amalgamato che serviva da asticella della bottiglia. La scarica esterna si eccitava per mezzo dello scaricatore $e e^{\prime}$, adoperandolo nel modo indicato nella figura.

Con l' apparato cosi modificato ripetei le esperienze dette di sopra ed i risultati furono affatto simili a quelli già indicati. E qui, per mostrare l' andamento del fenomeno, trascrivo nello specchio seguente $i$ risultati di alcune esperienze eseguite con la bottiglia ad armature di stagnola ('): ed essi

(1) Le esperienze fatte con Ja bottizlia ad armatura interna di mercurio saranno esposte in seguito ed a parte; perciò le ricerche si intendono eseguite con le bottiglio ad annature di stagnola quando non sia specificato che s'adoperò quelia n mercurio. 
sono le medie di 5 o più misure fatte per ciascuna condizione di esperienza.

TABELLA I.

\begin{tabular}{|c|c|c|c|}
\hline \multirow{2}{*}{ CARICHE } & \multicolumn{2}{|c|}{ SPOSTAMENTO DELL' INDICE } & \multirow{2}{*}{$\begin{array}{c}\text { MEDIF DI } \\
\mathrm{C}\end{array}$} \\
\hline & $\begin{array}{c}\text { Istantaneo } \\
\text { C }\end{array}$ & Residuo & \\
\hline $\begin{array}{l}1 \\
2 \\
3 \\
4 \\
5\end{array}$ & $\begin{array}{c}0,0 \\
\text { incerto } \\
0,5 \\
1,6 \\
3,9\end{array}$ & $\begin{array}{l}0,0 \\
0,0 \\
0,0 \\
0,35 \\
1,0 \text { circa }\end{array}$ & $\begin{array}{l}0,0 \\
0,0 \\
0,45 \\
1,50 \\
\mathbf{3 , 5}\end{array}$ \\
\hline $\begin{array}{l}5 \\
4 \\
3 \\
2 \\
1\end{array}$ & $\begin{array}{c}3,1(1) \\
1,4 \\
0,4 \\
\text { incerto } \\
0,0\end{array}$ & $\begin{array}{l}0,8 \text { circa } \\
0,2 \\
0,0 \\
0,0 \\
0,0\end{array}$ & \\
\hline
\end{tabular}

La tabella precedente è disposta in modo che si comprende facilmente; ed i numeri trascritti indicano che al momento della scarica l'aria del termometro subisce una dilatazione. per cui l' indice di esso si abbassa: la quale dilatazione impercettibile per le piccole cariche, cresce rapidamente col crescere di queste. Si scorge inoltre che detta dilatazione rapidamente sparisce, e qualche volta una parte o residua (colonna III) si dilegua lentamente. Coteste dilatazioni sembrano essere prodotte dal calore svolto nella bottiglia, e generato da quelle scintille che si veggono nel buio a mo' di arborescenze guizzare dai bordi liberi delle armaturs e correr su per le superficie verniciate del vetro. Le quali scintille riscaldando, forse, l' aria ed il retro son la cagione che parte della dilatazione sparisce rapidamente e parte lentamente: e concordemente alle dilatazioni, esse sono breri,

(1) Questa seconda serie fu eseguita dopo arero introdotto nel canncllo termometrico una colonna d'alecol circa tripls di quella adoperata nol caso precadeate e nerciò gli spostamenti prodotti furono più piccoli. 
esili e poco luminose quando la carica della bottiglia è piccola; e crescono poi in modo regolare e rapidissimo al crescere delle cariche, mostrando così che la scarica interna cresce col crescer di quelle (').

Queste osservazioni parmi dimostrino chiaro, come già avero supposto, che scaricando una bottiglia in opportune condizioni, oltre la scarica ordinaria se ne prodica un'altra nel suo interno; per distinguere le quali chiamerò scarica esterna la prima e scarica interna la seconda.

Dopo cid provai diversi modi a fine di aumentare la scarica interna; e la maniera più facile per raggiungere questo scopo si fu quella di sperimentare, come si è detto, con cariche a potenziali molto elevati: nel qual caso la scarica interna s' accresce, e come è naturale, a detrimento del calore svolto della esterna. Ed invero, oltre il fatto accennato in principio di questo scritto, aggiungerò ancora come in alcuni casi studiati da me, il calore svolto dalla scintilla eccitatrice esterna cresce assai più rapidamente del potenziale d'una carica costante; ma appena cominciano vigorose le scariche interne l'aumento termico ha luogo presso a poco in proporzione del semplice potenziale. Da tutto ciò si comprende di leggieri che a voler stabilire le leggi delle scarich $\theta$ esterne bisogna sperimentare con bottiglie bene isolanti e debolmente

(1) Il Siensens W. (Fortschritte der Physik, B. 20. s. 442) ha osservato con 180 termoelementi, di forro ed argentana, masticiati fra due Jastre di vetro formanti condensatore, che il galvanometro a specchio a quelle riunito mostrava una corrente corrispondente al loro riscaldamento, quando il condensatore si caricava e scaricava più volte di seguito: o Ja depiazione cessava soltanto dopo più oro. Lo mie esperienze ai riferiscono a fenomeni d' una natura affatto diversa; essi si manifestano solo al momento delle scaricbe, e sono prodotti da quella parte della bottiglia prossima ai bordi delle sue armature. Ed invero cotaj fonomeni si osservarono ancora o presso a poco con eguale intensità adoperando una bottiglis la cui armstura esterns ora pressoch tutta fuori del tormometro, o J'interna ne ora separata da un disco di sughero, posto ad un centimetro o due sotto il hordo superioro dell'armatura interma medesima, e masticistovi diligentemente cosi da tenere l'aria. Credo intanto, che anche lo dilatazioni termometriche osserrate da me sieno prodotte da risealdamento; e perciò nel corso di questo scritto parlero indistintamente di calore o di dilaturioni termonetriche. 
caricate; perchè solo in tal caso, come si rileva dallo specchio I, la scarica interua è trascurabile (').

Una seconda maniera per aumentare la scarica interna mi venne suggerita dall'influenza grande, che ha la forma della estremità dell'eccitatore sulla produzione della scintilla. A me, e forse a molti altri fisici ancora, è occorso di notare che quando si scarica una batteria avvicinando la palla dell' eccitatore a quella della batteria, si produce una scintilla poco rumorosa ed invece essa riesce assai più energica $\theta$ più lunga se la pallina dell' eccitatore s'avricini ad un' asticella, o meglio ad un filo unito all' armatura interna. Oltre di che ho sempre notato, nelle mie esperienze termiche, che quando la scintilla scatta fra palline di 10 o $12^{\mathrm{mm}}$ il calore che produce è molto minore di quello che genera la scintilla che scatta fra punte. Questi fatti mi fecero supporre che analoga influ. enza dovesse avere la forma degli elettrodi sul valore della scarica interna; e perciò provai a scaricare la bottiglia $B$ ora arvicinando alla pallina $n$ la $e^{\prime}$ dell'eccitatore, ed ora invece arvicinandovi l'estremo $e^{\prime}$ terminato a punta; e le varie misure eseguite in condizioni perfettamente eguali mi mostrarono sempre che nel primo modo si verifica una scarica interna circa doppia di quella che ha luogo nella seconda maniera. Fra i molti valori ottenuti do i seguenti che confermano quanto ho detto; ed essi sono i risultati medi di 5 o 6 misure per ciascun caso.

\section{Tabelila II.}

\begin{tabular}{c|c|c}
\hline CARICHE & CALORE & SCARICA ES'IERNA \\
\hline 5 & 1,4 & Fra pallina e punta \\
5 & 2,7 & due palline \\
5 & 1,2 & pallina e punta \\
5 & 2,3 & » duo palline
\end{tabular}

(1) Questa quistione ed un'altra ancora rolativa alla natura delle bottiglie sara trattata con maggioro ampiezza in una mia Memoria di prossima pubhlicazione. 
Messo in evidenza questa influenza speciale delle palline, di aumentare cioè la scarica interna, volli osservare se la si poteva accrescere viemaggiormente facendo alla scarica esterna produrre due scintille, entrambi fra palline. Perciò riunii l'armatura interna della bottiglia ad una delle palline dello spinterometro, grosse 15,5 e quindi avvicinavo l'eccitatore comunicante con l'armatura esterna all'altra pallina dello spinterometro stesso: e così si producevano due scintille esterne. In tal modo operando, e variando anche la lunghezza della scintilla nello spinterometro fra $3^{\mathrm{mm}}$ e $6^{\mathrm{mm}}$, ottenni con tre cariche impartite alla bottiglia i seguenti dati, medi di 5 o 6 misure ciascuno.

\begin{tabular}{c|c}
$\begin{array}{c}\text { Lunghezza della scintilla } \\
\text { nello spinterometro }\end{array}$ & $\begin{array}{c}\text { Calore } \\
\text { dearica interna }\end{array}$ \\
\hline 0 & 3 \\
$3^{\mathrm{mm}}$ & 3,2 \\
5 & 3,8 \\
6 & 3,3 \\
0 & 3,4
\end{tabular}

I numeri dello specchio precedente mostrano che l'influenza delle due scintille esterna, ad aumentare la scarica interna, è piccola ed anche non del tutto sicura: perciò io mi limitai, per semplicità, nello studiare le scariche interne, di far produrre una sola scintilla alla scarica esterna.

Intorno alle dimensioni delle palline, dirò che avendo sperimentato con la pallina della bottiglia di $16^{\mathrm{mm}}$ e con quella dell' eccitatore, la quale fu successivamente di 19 e di $24^{\mathrm{mm}}$, ho osservato che era maggiore la scarica interna quando si adoperò la pallina di $24^{\mathrm{mm}}$. Quindi ho unita alla bottiglia una pallina di $52^{\mathrm{mm}}$, ed all' eccitatore una di $62^{\mathrm{mm}}$, ed avendo fra esse prodotto la scarica ho ottenuto dei risultati poco o punto diversi dai precedenti. Laonde pare che sia utile adoperare palline non molto piccole, per accrescere la scarica interna: ed essere poi inutile lo adoperarne delle molto grandi. Questi confronti furono fatti con una bottiglia 
ard armatura interna di mercurio, la quale era piì bassa dell'esterna di 5 a $7 \mathrm{~cm}$.

Circa alla forma della punta, dirò che è bene che essa sia acuminata ed allungata, giacchè avendo sperimentato con una bottiglia ad armatura di stagnola, (in cui l'interna era $506 \mathrm{~cm}$. più alta dell' esterna), trovai che la differenza deila scarica interna era assai piccola tanto so si scaricara la bottiglia fra due palline di $16^{\text {nm }}$ e $19^{\mathrm{mm}}$, quanto se la si scaricava fra una pallina di $16^{\mathrm{mm}}$ e la punta dell' eccitatore, la quale era a cono assai ottuso, delle dimensioni e forme della figura 2.

La diversa intonsita della scarica interna pel variare della forma degli elettrodi, oltre all' essere dimostrata dal calore, viene altresì resa manifesta dai fenomeni luminosi, che si scorgono nelle bottiglie all' istante della loro scarica. Infatti tanto io che il mio assistente, ing. Bracchi, abbiamo osservato che le scintille, le quali guizzano dai bordi delle armature, sono sensibilmente più lunghe e vigorose quando la scarica esterna si produce fra palline, che quando ha luogo fra una pallina ed una punta. Ed a me parve, che nel primo caso le scintille interne fossero di lunghezza circa doppia che nel secondo.

Questi ed altri fatti ancora, che verrò esponendo, parmi mostrino che la bottiglia nel momento della scarica non può considerarsi del tutto come inerte e passiva, ed invece essa mostra una certa somiglianza con la pila; essendochè in questa come in quella l'energia elettrica, al momento della scarica o produzione della corrente, si manifesta parte nell'elettromotore, bottiglia o pila, e parte nel circuito esterno o congiuntivo. E la partizione della scarica fra l'interno e l'esterno della bottiglia od elettromotore dipende, sia dalla sua natura, sia da quella del circuito esteriore; imperocchè variando (come si è visto) la forma degli elettrodi e variando (come si vedrà) la natura del circuito e la condizione dello bottiglie, varia altresì il rapporto fra l'energia delle due scariche interna ed esterna: laonde io feci ancora diversi tentativi per accrescere il valore delle scariche interne. 
Ed in prima sperimentai con due bottiglie scintillanti (le cui armature esterne erano fatte con quadratini di stagnola di circa $2 \mathrm{~cm}$. di lato e posti a qualche millimetro di distanza fra loro) le quali disposte nei consueti termometri, mostrarono i soliti fenomeni delle scariche interne. Con una di esse il riscaldamento parve più cospicuo del consueto, giacchè si produssero le seguenti dilatazioni:

CARICHE CALORE

$5 \quad 3,8$ media di 5 misure.

56 . la bottiglia si perforò internamente al term.

Queste esperienze non poterono essere ripetute essendosi rotta la bottiglia.

La seconda bottiglia resistette meglio alle prove, essendomi limitato a 4 cariche solamente, e così potei fare un confronto più esatto fra la bottiglia scintillante e la stessa bottiglia rivestita di nuovo esternamente con una stagnola continua e non interrotta.

I dati seguenti sono le medie di pì̀ misure eseguite con questa bottiglia preparata nei due modi indicati.

Tabelia III.

\begin{tabular}{c||c|c}
\hline \multirow{2}{*}{ CARICHE } & \multicolumn{2}{|c}{ CaLORE NELIA BOTtigilia } \\
& Scintillante & Non scintillante \\
\hline \multirow{2}{*}{1} & 0,2 & 0,00 \\
2 & 0,7 & 0,09 \\
3 & $\mathbf{1}, 2$ & 0,5 \\
4 & $\mathbf{1 , 9}$ & 2,13
\end{tabular}

Questi numeri mostrano una lieve diversità fra le scariche interne delle due bottiglie, ma non una vera e cospicua prevalenza del calore svolto dalla bottiglia scintillante su quella ordinaria, come sarebbesi per avventura potuto supporre. E la interpetrazione di questo fatto, in apparenza singolare, ricavasi dalle apparenze luminose che si osservano nelle due bottiglie. Così, tanto io che il mio assistente ab- 


\section{4}

biamo osservato, che mentre in una bottiglia ordinaria, caricata con 3 unità elettrometriche, le scintille ai bordi liberi delle armature erano di 2 a 3 centimetri di lunghezza, grosse e vivaci: quelle di una bottiglia scintillante, per carica eguale, erano di circa $1 \mathrm{~cm}$. di lunghezza, pallide, esili e quasi come capelli (secondo l'espressione del mio assistente). Laonde può dirsi che se il calore delle molte scintille, che al momento della scarica si producono nell' armatura interrotta d' una bottiglia scintillante, non è maggiore di quello che si svolge in una bottiglia ordinaria, ciò $\grave{e}$ dovuto alla sensibilissima diminuzione che soffrono nella loro estensione le scintille sui bordi superiori e liberi di quelle. Talmentechè può dirsi, che lo scintille nelle interruruzioni dell' armatura son prodotte a scapito di quelle che nascono ai bordi liberi di essa. Dato adunque una determinata carica, un certo circuito ed una data natura di bottiglia, non si riesce ad accrescerne la scarica interna con l'accrescere il numero delle scintille, imperocchè con esso pare che decresca il loro vigore.

Le esperienze riportate in principio della pag. 13 mostrano inoltre un fenomeno importante. Da esse si rileva come il calore interno, che era inferiore a $4^{\circ}$ divenne, $6^{\circ}$ al momento che la bottiglia, nell'istante della scarica, fu perforata in un punto che trovavasi internamente al termometro. Il quale aumento di calore, io credo debba attribuirsi a ciò, che col perforamento della bottiglia gran parte della scarica ha luogo attraverso il foro in essa prodotto. Tale spiegaziono viene convalidatia dal fatto che un'altra bottiglia, che produceva con la scarica interna $4^{\circ}$ a $5^{0}$ di calore nel caso ordinario, ne produsse soli 2 al momento che si perford in un punto fuori del termometro. I quali fenomeni hanno analogia con altri ancora studiati da me e che esporrò piì tardi.

Un secondo tentativo per accrescere la scarica interna lo feci, variando l' ampiezza dell' armatura interna delle bottiglie, affine di aumentare possibilmente sulle loro pareti la lunghezza delle scintille (1). Perciò eseguii le misure con

(1) Qui i bene ricordare come I'illustre Prof. Rossetti ottenne dolle lunghe 
due bottiglie ad armature esterne eguali: in una però l'armatura interna si estendeva ad eguale altezza della esterna, ed in un'altra per oltre $7 \mathrm{~cm}$. di più. Queste bottiglie apparecchiate al modo consueto dettero i seguenti risultati medi di pì̀ misure.

TABeLla IV.

\begin{tabular}{|c|c|c|}
\hline \multirow{3}{*}{ CARICHE } & \multicolumn{2}{|c|}{ BOTTIGLIA AD ARMATURE } \\
\hline & Egu.uLI & Disuguali \\
\hline & $\begin{array}{l}\text { Calore } \\
\text { C }\end{array}$ & $\begin{array}{c}\text { Calore } \\
\text { C. }\end{array}$ \\
\hline $\begin{array}{l}4 \\
5\end{array}$ & $\begin{array}{l}1,2 \\
2,5\end{array}$ & $\begin{array}{l}1,3 \\
2,0\end{array}$ \\
\hline
\end{tabular}

I valori precedenti, relativi al riscaldamento interno delle due bottiglie, mostrano evidentemente che il calore in entrambi generato. per determinate quantità di elettricità, è indipendente dall' ampiezza delle armature, salvo piccola differenza. Ma queste misure essendo state eseguite con bottiglie e termometri diversi non mi parvero meritevoli $\mathrm{di}$ piena fiducia ed atte a risolvere la quistione.

Per la qual cosa rolli più accuratamente e completamente riprovare codesta influenza : e percio sperimentai con una sola bottiglia di vetro verde sottile, che conservava bene le cariche. Essa esternamente, per due terzi, era coperta di stagnola, e fu successivamente rivestita internamente con stagnola avente diverse altezze : il che facilmente potei praticare, avendo alla canna termometrica $\mathrm{TT}^{\prime \prime}$ masticiato in $\mathrm{T}$ una ghiera d'ottone a vite. la quale s'apriva agevolmente e permetteva di modificare variamente l'armatura interna della bottiglia. I risultati medi di 5 o più misure sono i seguenti:

seintille scaricando un condensatore ineompleto, fatto d' una lastra di vetro ricoperto su una sola sua faccia di stagnola, l'altra essendo naturalmente rivestita più o meno d' umidità. Veggasi la Memoria sulla forma di queste scintille nel $\mathrm{N}$. Cimento, vol. VII ed VIII, pag. $33,1872$. 
TABELLA V.

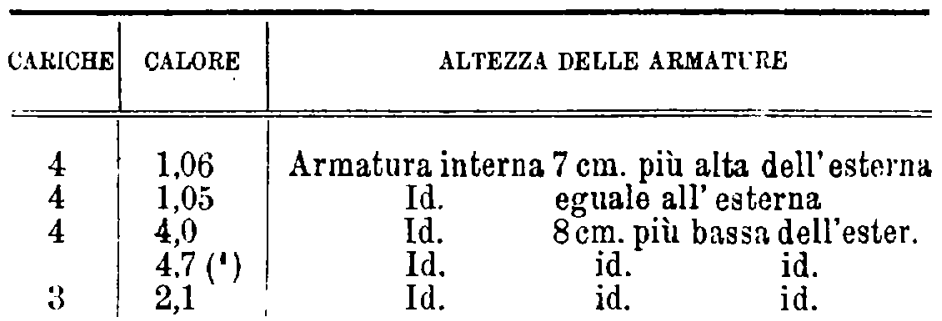

I dati precedenti mostrano con ogni certezza, che per una data altezza di armatura esterna:

$1^{\circ}$. La scarica interna della bottiglia è presso a poco costante ed invariabile, sia quando l'armatura interna è piư estesa, sia quando è di eguale altezza della esterna;

$2^{\circ}$. La scarica interna invece è quasi più del quadruplo di quella che si verifica nei casi precedenti, quando l'armatura interna è sensibilmente piì piccola $(8 \mathrm{~cm}$. più bassa) dell' esterna.

La spiegazione, di questo modo singolare di agire dell' armatura interna diversamente ampia, parmi possa essere la seguente. Quando detta armatura si scorcia così da divenire sensibilmente più bassa e più piccola dell'esterna, il condensatore noterolmente diminuisce d'estensione, il potenziale in esso (a parità di carica) aumenta e con esso deve aumentare la scarica interna; la quale viene di certo agevolata dall' estensione maggiore dell' armatura esterna, che quasi eccita la scarica interna ad estendersi dal bordo dell'una a quello dell' altra stagnola: percio in tale bottiglia si svolge più calore che in quella nella quale le armature sono eguali. Che il potenziale sia realmente aumentato nel caso precedentemente contemplato, oltre che dalla diminuita esten-

(1) Le asperienze con questa bottiglia non poterono prolungarsi giacchè essa alla terza misura ( con l'arratura interna $8 \mathrm{~cm}$. più bassa dell' esterna e con $4 \mathrm{ca}-$ riche) si perforò : ma siccome avevo preveduto il caso cosi sperimentai dapprima o piu volte con 3 sole cariche, $\theta$ trovai in media che il calore prodotto in questo caso fu di 2,1 cioè il doppio di quello svolto nolla stessa bottiglia ad armature eguali u caricata con 4 unitia. 
sione dell' armatura, viene dimostrato chiaramente dal fatto che la bottiglia ad armatura interna piccola fu perforata con le 4 cariche, che benissimo areva sostenute prima, e quando l'ar'matura interna era più estesa.

Quando poi la stagnola interna s' estende fino alla stessa altezza dell' esterna, allora il potenziale decresce e cou esso la scarica interna: e talo ultima diminuzione tanto più facilmente ha luogo, inquantochè le armature essendo eguali non vi è alcuna tendenza a diffondersi della scarica sulla superficie della bottiglia. In questo caso adunque il calore che si produce, deve essere ed è in effetti minore di quello svolto nel caso precedente.

Finalmente quando l'armatura interna s'estende più dell' altra, il potenziale seguita a decrescere e con esso dovrebbe altresi decrescere la scarica interna. Perd la stessa maggiore estensione della stagnola facilita dall' altro canto la scarica, essendochè essa viene quasi eccitata ad estendersi sul vetro interposto fra $\mathrm{i}$ due bordi liberi delle due armature. Le due influenze sono adunque opposte e possono, dentro certi limiti compensarsi, o l' una ecceder l'altra; e perciò potrà accadere che la boltiglia ad armatura interna più estesa dell' esterna produca una quantita di calore eguale, maggiore o forse minore di quella che produce la bottiglia ad armature eguali. Il primo caso si verificò con la bottiglia detta di sopra, (vedi $1^{a}$ e $2^{a}$ esperienza nella tabella V) ad armatura interna piu estesa dell'esterna; ed il secondo si è manifestato con una bottiglia ad armatura interna di mercurio, come diro in seguito. Che poi la ineguale estensione delle due stagnole sulle bottiglie agevoli la produzione delle scariche interne, può anche rilevarsi dal fatto, che quando si carica una di codeste bottiglie, si manifestano spesso dello scariche parziali sulla sua superficie, accompagnate da luce e calore; ed il fenomeno è assai più distinto quando l'armatura interna è più piccola dell' esterna. Invece un tal fenomeno manca, a parità di coudizioni, con la bottiglia ad armature uguali.

Questa interpretazione viene inoltre confermata dai fenu- 
meni luminosi che si manifestano nelle bottiglie. Quanio la loro armatura interna è più piccola dell'esterna, al momento della scarica una viva luce quasi continua s'espande dal bordo dell'armatura interna a quello dell' esterna. Quando le armature sono eguali le scintille che dai bordi si sollevano sono fitte, vivaci, brevi, e regolarmente crescono con le cariche. Invece nel caso dell' armatura interna più estesa noi osserviamo che gli sprazzi di luce fra i bordi delle due armature crescono rapidamente, così da estendersi subito dall'uno all' altro: e quindi aumentano poi più lentamente. Esse sono vivaci e di luce bianca in basso, violetta in alto ore si ramificano. ì da avvertirsi che queste scintille, nel caso dell' armatura interna più estesa, sono più rade (nelle mie esperienze erano forse ad un centimetro di distanza l'una dall'altra) di quelle che si osservano nelle bottiglie ordinarie, nelle quali dette scintille sono assai fitte ed a 304 millimetri di distanza fra loro: perciò quelle abbenchè più lumghe pure producono lo stesso calore di queste.

Dopo aver variamente modificato le condizioni delle bottiglie, variai ancora quelle del circuito esteriore: e perciò studiai l'influenza della forma degli estremi degli elettrodi, la quale produsse quella serie di fenomeni gia descritti in principio di questa Memoria. Poscia modificai la resistenza del circuito esterno, interponendo nell'arco scaricatore una colonna d'acqua che variai in lunghezza da 4 a $28 \mathrm{~m}$. ('); ed osservai che allora il calore della scarica interna decresceva a mano a mano che la resistenza esteriore aumentava: in maniera che quando questa era formata da una certa lunghezza di colonna d'acqua, il calore della scarica interna diveniva insensibile, non muovendo punto l'indice termometrico.

In quanto ai fenomeni luminosi, che si osservano nelle bottiglie diro come osservai, che col crescere della resistenza esterna le scintille interne diminuivano di numero, di viva-

(1) L'acqua adoperata era di fonte, o riempiva dei tubi di vetro vernioiati estervamente, ripiegati in forma $\mathrm{di} \mathrm{l}$, Juvæhi ciascuno circa $4 \mathrm{~m}$, e 0,005 di vanu inleruo. 
cità e di estensione sin quasi a divenire invisibili. E per meglio studiarlo adoporai una bottiglia ad armatura interna più alta dell' esterna: e vidi che col crescere della resistenza esterna, le scintille si riducevano ad una o due soltanto, ed apparivano più sottîli e meno luminose dell' ordinario.

Quando si adoperano bottiglie ad armatura interna di mercurio Ia scarica interna par che si accresca. Così avendone adoperata una nella quale l'armatura interna di mercurio era di circa $10 \mathrm{~cm}$. più bassa della esterna, trovai che in essa la scarica interna era assai vigorosa e certamente superiore a quella che si verifica in bottiglie simili, ad armatura di stagnola. Qui in seguito sono riportati i risultati di due serie di esperienze, e ciascun valore di esse serie corrisponde alla media di 506 misure:

TabelLa VI.

Bottiglia ad armatura interna di mercurio.

\begin{tabular}{c||c|c||l}
\hline \multicolumn{1}{l||}{} & \multicolumn{2}{c||}{$\begin{array}{c}\text { DEVIAZIONI DELL' INDICE } \\
\text { CARICHE }\end{array}$} & Rosidue \\
& $\begin{array}{c}\text { Istantanee } \\
\text { C }\end{array}$ & & OSSERVAZIONI \\
\hline \hline 5 & 10,6 & 5,3 & Scarica esterna fra palline \\
4 & 8 & 3,7 & \\
3 & 4,5 & 2,6 & \\
2 & 2,3 & 1,3 \\
1 & 0,15 & 0,0 \\
1 & 0,4 & 0,0 \\
2 & 2,0 & 1,0 & \\
3 & 4.0 & 1,7 & \\
4 & 7,7 & 4,0 & \\
5 & 10,3 & 5,3 &
\end{tabular}

Questi numeri mostrano che la scarica interna in questa bottiglia è 203 volte superiore a quella ottenuta nei casi analoghi precedenti. Tuttavia per assicurarmi meglio del fatto, ed avendo considerazione alla grande influenza che esereita l'ampiezza delle armature, rifeci due serie di esperienzo 
di confronto. Nella prima adoperai noo stesso termometro ed una medesima bottiglia, una volta con l' armatura interna di mercurio ed una seconda con l'armatura interna di stagnola, le quali erano della stessa altezza fra loro, e circa $9 \mathrm{~cm}$. più basse della esterna. Le misure furono più volte ripetute, la carica fu costantemente di 3 unità, la scarica si eccito fra palline di 19 e $24^{\text {rom }}$ ed i risultati medi sono i seguenti :

CALORE

4,6 Bottiglia ad armatura interna di mercurio

$3,1 \gg \quad$ stagnola

In una seconda serie poi adoperai del pari una stessa bottiglia ed un medesimo termometro; $\theta$ per esser sicuro di nulla rariare, sperimentai prima con la bottiglia ad armatura interna di stagnola, $7 \mathrm{~cm}$. più bassa dell' esterna: quindi versai pel tubo $t$ del mercurio nella bottiglia. fiuo all'altezza alla quale giungeva la stagnola interna e poscia per un centimetro ancora, ed i risultati medi di 50 più misure, eseguite in modo perfettamente simili sono qui sotto registrati:

CARICHE CALORE

TABELLA VII.

$3 \quad 3,1$ Armat. inter. di stagnola $7 \mathrm{~cm}$. yiù bassa dell'est.

$36,05 \gg$ di mercur. $7 \mathrm{~cm}$. più bassa dell'est.

$35,4 \gg$ di mercur. $6 \mathrm{~cm}$. più bassa dell'est.

Queste varie misure mostrano con tutta evidenza, che il calore della scarica interna d' una bottiglia a mercurio è superiore sensibilmente a quello svolto da una bottiglia eguale ad armature di stagnola. Si osserra altresì nell'ultimo quadro, che col crescere dell' estensione dell' armatura interna a mercurio decresce la scarica interna.

Quest'ultima osservazione mi spinse a studiare l' effetto dell'estensione dell' armatura di mercurio sulla scarica interna: e percio adoperai uno stesso apparecchio, o nella bottiglia che ne faceva parte rersavo diversa quantita di mercurio, quindi sperimentai al modo consueto, ed i risultati medi di più misure sono i seguenti : 
CALORE

3,8 Armatura interna 5 o $6 \mathrm{~cm}$. piu bassa dell' esterna

$1,4 \quad \gg \quad$ eguale all' altezza dell'esterna

$2,8 \quad \gg 5 \mathrm{~cm}$. più alta dell' esterna.

Questi dati mostrano che il calore massimo si ha quando l'armatura interna è più picccla; il che concorda con cid che precedentemente si è detto, parlando delle bottiglie con stagnola. Nel caso che l'armatura interna di mercurio sia più alta della esterna, il calore svolto è maggiore di quello che si produce con bottiglia ad armature eguali: il quale fatto fu preveduto e discusso quando si parlo dell' influenza dell'estensione delle armature di stagnola ('). E finalmente pare altresì che anche ad armature eguali l' effetto termico della bottiglia a mercurio sia superiore a quello delle bottiglio ordinarie. Pure per dimostrare ció con sicurezza, misurai il calorico srolto da una stessa bottiglia, prima ad armatura interna di stagnola e poscia di mercurio, aventi sempre la stessa altezza della esterna, ed ottenni $\mathrm{i}$ dati medi seguenti, relativi ad una carica costante di 3 unità elettrometriche.

\section{CALORE}

0,4 Armatura interna di stagnola $1,47 \gg$ di mercurio.

Laonde si rilera, che a parità di condizioni, l'armatura interna di mercurio produce sempre nelle bottiglie una scarica maggiore che non faccia l'armatura interna di stagnola ( $)$.

A completare da ultimo lo studio delle bottiglie con mercurio, mi parve utile di osservare ancora l' influenza che aveva la forma degli elettrodi nel promuover la scarica esterna; e ripetuto le solite esperienze osservai, come di consueto,

(1) Yedi pag. 17 o 1. e 2. esperienza della Tabella $\mathrm{V}$.

(2) Su queste dilatazioni temometriche ottenute con la bottigjis ripien di mercirio, può forso influire la diminuita massa d'aris del termometro, imperocchò le dijatazioni in questo casn divontano più mpide e percio le pendito di calore mono sepsibili. 
che quella è assai più cospicua, quando la scintilla esterua si produce fra palline, che quando la si eccita fra punta e pallina. I dati seguenti, medi di più misure, che si riferiscono ad una bottiglia ad armutura interna di mercurio di circa $10 \mathrm{~cm}$. più bassa dell' esterna, confermano questo fatto:

CARICHE CALORE

$\begin{array}{crcc}5 & 10,3 & \text { Scarica esterna eccitata fra palline } 16 \text { e } 24^{\mathrm{mm}} \\ 5 & 5,2 & » & 16^{\text {nm }} \text { e punta } \\ & & & \text { agnzza. }\end{array}$

In quanto ai fenomeni luminosi che si manifestano con la bottiglia ad armatura interna di mercurio, dirò che essi presentano analogia completa con quelli osservati nelle bottiglie ad armatura interna di stagnola.

Dopo di aver dimostrato l'esistenza di codeste scariche interne delle bottiglie è necessario di cercarne la spiegazione; la quale parmi possa essere la seguente.

Quando si carica un condensatore qualunque, come un quadro di Franklin, sappiamo che le due armature prendono opposte elettricità, s'influiscono reciprocamente e danno luogo alla condensazione elettrica ed alla carica dell'apparecchio. Codeste elettricità inoltre, io credo che debbano agire per influenza sulla zona di vetro che le circonda, ed operarvi in modo da indurre su ciascuna delle facce una zona neutra intorno a ciascuna armatura, e quindi un'altra zona carica d'elettricità opposta a quella della corrispondente armatura. Al momento della scarica, l' elettricità della zona elettrizzata, con parte di quella dell'armatura si neutralizza; le scintille corrono sulle zone neutre del vetro e la scarica interna si produce.

Per dimostrare l'esistenza di questi stati elettrici dei condensatori io mi son servito di quadri di Franklin con armature sia circolari, sia rettangolari, sia anche di altra forma; ed il vetro di essi, alcune volte era verniciato altre volte terso e secco. Caricavo debolmente questi quadri, affinchè scariche superficiali non si producessero, e poscia sostenuti in posizione quasi verticale vi soffiavo sulle due facce, 
con un mantice, la nota miscela di solfo e minio in polvere. E con tale artifizio subito si formavano delle figure elettriche assai distinte, bellissime ed istruttive.

Una delle armature si ricopriva, per es. di solfo, ed era circondata $\theta$ come incorniciata da una zona di vetro affatto priva di polrere; la quale poi a sua volta rimaneva chiusa e inviluppata da un'altra zona ricoperta da polvere di minio. Dal che si conclude, che l'armatura in discorso era carica d' elettricità positiva, che la prima zona del vetro, era allo stato naturale e che la seconda era carica di elettricità negativa. Le figure ed i fenomeni sono analoghi sull'altra faccia, salvo che gli stati elettrici che vi si riscontrano sono opposti a quelli più sopra indicati.

Se si carica il quadro vigorosamente, allora sulla superficie del vetro si formano delle scintille che accusano delle scariche parziali, che complicano oltre modo lo stato elettrico delle superficie del vetro; per lo che con le note polveri vi si formano delle figure elettriche assai complesse $\theta$ spesse volte delle arborescenze assai eleganti che indicano il luogo ove si produssero le scintille. Nè meno complesso ed intricato è lo stato elettrico del quadro dopo che lo si è scaricato nel modo ordinario; inquantochè il vetro riman carico in modo irregolare; e conserva questo stato per molte ore e non può adoperarsi, per riottener le figure, prima che non sia del tutto ritornato allo stato naturale.

Le bottiglie si devon comportare come i quadri di Franklin; ed avendone adoperate due con bordo rivestito di ceralacca, chiuse con tappi di sughero, ed avendole caricate con opposte elettricità vidi, soffiandovi le polveri, che l'armatura esterna dell' una si ricopriva di solfo; mentre che la parte superiore del vetro, il tappo e l'asticella si coprivano di minio; nell' altra bottiglia il minio ricopriva la stagnola ed il solfo rivestiva il retro verniciato; e rimaneva fra l'armatura e la regione elettrizzata, la zona neutra ordinaria. Noterò che sull' armatura comunicante col snolo poca polvere si raccoglieva. Nè voglio mancare di dire che con questi studi, ripetuti e variati su condensatori di diverse specie di vetro, e 
coperti da differenti vernici, si potrd forse ricavare qualche utile cognizione intorno alla varia natura dei coibenti, che si adoperano nella costruzione dei condensatori, ed anche intorno al modo diverso d'agire e diffondersi delle due elettricità sui coibenti.

Forse la formazione di queste zone elettrizzate non è un fenomeno tanto semplice come s'è detto in principio, giacchè io credo che a produrle concorrono contemporaneamente entrambi le armature. Ciascumo di esse infatti produce sulla corrispondente faccia di vetro la rispettiva zona elettrizzata, la quale poi influisce sull'altra faccia ed insieme all'altra armatura dà luogo ad uno stato elettrico opposto. Così si potrebbero forse paragonare, in questo caso, le due armature ai poli di una calamita a ferro di cavallo, la zona neutra alla regione di contatto fra essi poli e l'ancora, e questa sarebbe, nel caso del condelsatore, rappresentata dalla zona di vetro carica sulle due sue facco d'elettricità oppostia a quella delle due armature.

Queste zone poi spesso sono più o meno modificate dalla conducibilità elettrica propria del coibente, forse diversa per le due elettricita per cui si osserva come una specie di espansione del fluido delle armature sul retro che le circonda, resa anche più energica da punte che possono riscontrarsi sui bordi delle armature istesse; ed in genere non è difficile che il fenomeno varii più o meno per moltissime cagioni e specialmente se si opera con quadri ad armature ineguali coine si dirà qui in seguito.

Per eseguire gli studi su codesti condensatori ho costruito dei quadri con armature di forma circolare di diverso diametro, ed appiccicate sulle due facce della lastra, cosi che $\mathrm{i}$ loro centri si trovavano sopra la stessa normale alla lastra medesima. Sperimentando con questi quadri alla maniera consueta si scorge, quando la differenza delle armature non sia molto grande, che la zona neutra che circonda la piccola arinatura si estende dal suo bordo a quello della grande, ed in corrispondenza del bordo di questa si manifesta la zona elettrizzata. Quando la differenza dei diametri cresce, la zona 
neutra si estende ancora, ed io ne ho con un quadro ottenuta una di $4,5 \mathrm{~cm}$. di larghezza, essendo una delle armature di 10 e l'altra di $19 \mathrm{~cm}$.: ed in tal caso anche la zona elettrizzati sul vetro apparisce generalmente più ampia ed estesa. Al dj là poi dell'armatura maggiore o sulla faccia corrispoudente del vetro si forma la consueta zona neutra e quindi quella d'opposta elettricità di essa armatura.

Da tutto cio si comprende come le scintille, nei condensatori ad armature ineguali, possono accrescersi in alcuni casi e percio produrre delle scariche interne più energiche: sulle quali influiscono e potentemente, come si disse, la diversitì del potenziale che un condensatore acquista per una data carica, quando si fa variare la grandezza di una delle due armature: ed i fenomeni debbono allora riuscire più complessl.

Un fenomeno deguo di nota osservai coll questi condensatori ad armature ineguali, ed è quello dell' inversione della carica. Cosi avendone caricato uno, le cni armature avevano i diametri di 10 e $19 \mathrm{~cm}$. osservai che la piccola armatura s'era ricoperta di minio e perciò era negativa: intorno ad essa ed a circa $4,5 \mathrm{~cm}$. dal bordo vi era irregularmente la zona ricoperta di solfo. Quindi scaricai il quadro, ne ripulii la faccia coperta dalla piccola armatura, vi soffiai di nuoro il consueto miscuglio, ed osservai che essa armatura si ricoprì di solfo, ossia essa aveva acquistata carica positiva e per conseguenza opposta alla primitiva. La piccola armatura era circondata da una breve zona neutra e poscia seguiva una zona negativa netta, regolarissima, finamente dentellata coperta completamente di minio e situata fra $i$ bordi dello due armature. La inversione della carica adunque avrenuta nell'armatura, dopo la scarica scorgevasi chiaramente; e tanto più inquanto che l'armutura opposta, non essendo in alcun modo stata toccata nella seconda parte dell' esperienza, rimaneva sempre coperta di solfo come lo era aranti la scarica: così che entrambe le armature erano coperte di solfo. Le figure elettriche pel fatto delle inversioni possono essere più 0 meno svariate e complesse a seconda delle circostanze: ed io ne ho ottenuto delle singolarissime ed assai belle. 
In quanto poi alla natura di queste scintille interne diru the esse mi sembrano potersi considerare come una specie di scintille eccitatrici, sui generis, che prendono vita $\theta$ calorico a spesa della vera e propria scintilla eccitatrice esterna, percui questa perde di energia col crescere di quelle. Ed è quindi alla loro formazione ed al loro rapido incremento, che debbonsi ascrivere buona parte delle irregolariti che si manifestano nel calore svolto dalla scintilla eccitatrice esterna, quando si sperimenti con cariche a potenziali molto elevati.

Ed ora, prima di metter termine a questo mio scritto parmi utile, per aiutar la memoria del lettore, di dare il seguente

Riassunto: Quando si scarica una batteria fortemente carica, si produce in esso un rumore sordo, caratteristico e come un tonfo; il vetro delle bottiglie ai bordi delle armature s'illumina vivamente, ed in esse si svolge calore; infatti avendo introdotta una di codeste bottiglie in un appropriato termometro ad aria, osservai che questa si dilatava nel momento della scarica.

Dal che risulta che oltre la ordinaria scarica esterna della bottiglia se ne verifica un'altra nel suo interno, che per distinguerla ho chiamata scarica interna: ed essa ha luogo lungo le pareti del condensatore, non ricoperte dalle rispettive armature, e si rileva dalla luce $e$ dal calorico che si srolgono nella bottiglia al momento della scarici sua. Talmentechè l'elettricità d'un condensatore, nel momento della scarica, si neutralizza parte all'interno e parte all'esterno di esso. E misurando la scarica interna dalle dilatazioni termometriche che produce si perviene alle seguenti conelusioni :

$1^{\circ} \mathrm{Il}$ calore svolto dalla scarica interna è trascurabile o nullo con le deboli cariche, peró oltre un dato limite si appalesa e cresce rapidissimamente con le cariche istesse, a scapito, naturalmente, di quello che si svolge con la scintilla eccitatrice esterna: un primo mezzo adunque per accrescere codesto calore o scariche interne è quello di sperimentare con bottiglie cariche ad clevato potenziale: 
$2^{\circ}$ La scarica interna inoltre creste in modo assai sellsibile se si eccita l' esterna, facendo scattare la scintilla fra due palline di 20 a $30 \mathrm{~mm}$ di diametro, $\theta$ diminuisce invece, fin circa alla metà, se la si eccita fra una punta ed una delle indicate palline.

L' inverso si verifica pel calore dovuto alla scintilla eccitatrice esterna;

$3^{\circ}$ La scarica interna cresce per una data carica, se si diminuisce l'armatura interna della bottiglia. Essa scarica decresce con l'aumentare di detta armatura fino a che raggiunge l' estensione dell' armatura esterna, da indi in là rimane presso a poco indipendente dalla sua ampiezza, nei limiti delle mie esperienze. La cagione di questi fenomeni è complessa : essi dipendono in parte dal variare che fa il potenziale della carica con l'estensione dell'armatura, ed in parte ancora dall' influenza che esercita la diversa ampiezza delle due armature sul numero ed energia delle scintille interne;

$4^{\circ}$ La scarica interna è la stessa sia adoperando una bottiglia ordinaria sia adoperandone una scintillante;

$5^{\circ}$ La scarica interna, decresce fino a zero quando si accresce molto la resistenza del circuito esteriore;

$6^{\circ}$ La scarica interna, a parita di condizioni e forse più energica quando s' adopera una bottiglia ad armatura interna di mercurio. In tutto il resto questa bottiglia si comporta come una ordinaria ad armature di stagnola.

Tutte queste conclusioni ricarate dalle dilatazioni termometriche vengono pienamente riconfermate dai fenomeni luminosi, che si manifestano nelle bottiglie: essendochè la vivacità ed estensione delle scintille interue corrispondono esattamente e sempre all' intensità delle dilatazioni termometriche;

$7^{0}$ Queste scariche interne son dorute, io credo, a cho ciascuna armatura induce o risveglia nel coibente che le circonda una zona carica di elettricità opposta alla propria, la quale è separata dall' armatura corrispondente da un'altra zona di vetro allo stato naturale. Al momento della scarica 
parte dell' elettricità dell' armatura e della zona elettrizzata si neutralizza con produzione di scintille e calore, e quindi della scarica interna;

$8^{\circ}$ Tali zone elettrizzate sono rese manifeste da elegantissime figure elettriche che si producono soffiando su dei quadri di Franklin di vetro verniciato, (al limite delle armature) o meglio di ebanite, 0 sulle bottiglie di Leyda il noto miscuglio di solfo e minio;

$9^{6}$ Quando i quadri sono ad armature ineguali. la zona neutra dalla parte dell'armatura piccola, non che la zona elettrizzata, aumentano d' estensione, e perciò nel momento della scarica le scintille su di codesti condensatori debbono allungarsi, rinvigorirsi ed aumentare la scarica interna, in alcuni casi almeno;

$10^{\circ}$ Cou questo metodo, studiando dei quadri di vetro ad armature ineguali o meglio dei quadri di ebanite ad armature ineguali od eguali, ho osservato che dopo avere scaricato codesti quadri, nel modo ordinario, le armature, mostravansi cariche d'elettricita opposta a quella che averano originariamente.

E forse questo metodo di ricerche, più ampiamente modificato ed esteso potrà dare delle ulteriori ed utili indicazioni sulla stessa inversione delle cariche, sulla natura dei coibenti e vernici che si adoperano nei condensatori, sul vario modo di espandersi delle due elettricità sui coibenti ec.; intorno alle quali cose darò forse in appresso più ampie indicazjoni. 